\title{
Avaliação do consumo de bebida alcoólica na dieta habitual de pessoas que vivem com HIV/AIDS em unidade de referência da cidade Rio de Janeiro
}

\section{Evaluation of alcohol consumption in the usual diet of people with HIV/Aids in a reference unit of the city of Rio de Janeiro}

\author{
Camila Bezerra Gomes de Lima ${ }^{1}$ \\ Paulo Roberto Borges de Souza Júnior ${ }^{2}$
}

Segundo o Ministério da Saúde, no Brasil, de 1980 a junho de 2018, foram identificados 926.742 casos de AIDS. Nos últimos cinco anos o país tem registrado, anualmente, uma média de 40 mil novos casos de AIDS. A introdução da terapia antirretroviral (TARV) para o tratamento de pes-soas com HIV/AIDS ocasionou redução da morbidade e aumento da sobrevida dos casos de AIDS, bem como melhora da qualidade de vida em pessoas vivendo com HIV/AIDS (PVHA). Em contrapartida, entre as PVHAs houve um aumento do consumo de substâncias que causam dependência e repercutem na vida social, econômica e psicológica dessa população. O uso de álcool por PVHA está relacionado a um pior prognóstico, com aumento da incidência de comorbidades, como a coexistência de doenças hepáticas e anemia.

Objetivos: Avaliar consumo de bebida alcoólica (cerveja) e relação com perfil sociodemográfico de PVHA.

Metodologia: Estudo de corte transversal, com amostra de 69 indivíduos assistidos em um hospital de referência para o tratamento de HIV/AIDS na cidade do Rio de Janeiro, utilizando-se de Questionário de Frequência de Consumo Alimentar (QFCA) para levantamento de informações sobre a dieta habitual.

Resultados: Acerca do consumo de bebidas alcoólicas e suas possíveis correlações, observou-se a participação da cerveja com um consumo semanal representativo em meio aos indivíduos do sexo masculino (34,1\%), com primeiro grau incompleto (40\%), renda familiar estimada entre $1 \mathrm{e}$ 3 salários mínimos (30\%) e moradores da Zona Norte (33,3\%) do município do Rio de Janeiro. O perfil sociodemográfico de indivíduos que fazem consumo diário de cerveja mostra que a escolaridade exerce influência sobre as decisões de consumo desses indivíduos, uma vez que os que possuíam mais anos de estudo abdicavam, em sua maioria, desse hábito, onde é possível inferir que há um maior grau de apropriação do conhecimento acerca dos efeitos adversos gerados pelo consumo de álcool durante o tratamento com a terapia antirretroviral.

Conclusão: Diante de tais resultados, fica clara a necessidade da criação de um espaço de diálogo em que se destaque a Educação Alimentar e Nutricional (EAN), a fim de que esses indivíduos sejam aconselhados a cerca das adversidades do uso contínuo de bebidas alcoólicas e o que estas acarretam ao tratamento do HIV/AIDS, trazendo-os assim, ao papel de protagonistas do cuidado com a própria saúde. 
Palavras-chave: HIV; dieta habitual; cerveja; QFCA.

Keywords: HIV; usual diet; beer; QFCA.

1 Mestranda em Informação e Comunicação em Saúde no Instituto de Comunicação e Informação Científica e Tecnológica em Saúde da Fiocruz (PPGICS/ICICT/Fiocruz).

2 Pesquisador do Laboratório de Informação em Saúde do Instituto de Comunicação e Informação Científica e Tecnológica em Saúde da Fiocruz (LIS/Fiocruz). 